\title{
Fast in-database cross-matching of high-cadence, high-density source lists with an up-to-date sky model
}

\author{
B. Scheers ${ }^{\text {a,* }}$, S. Bloemen ${ }^{\text {b,c }}$, H. Mühleisen ${ }^{a}$, P. Schellart ${ }^{\text {d,b }}$, A. van Elteren ${ }^{e}$, M. Kersten ${ }^{\text {a }}$, \\ P.J. Groot ${ }^{\mathrm{b}}$ \\ a CWI-Centrum Wiskunde E Informatica, PO Box 94079, 1090 GB Amsterdam, The Netherlands \\ b Department of Astrophysics, IMAPP, Radboud University, 6500 GL Nijmegen, The Netherlands \\ ${ }^{c}$ NOVA Optical InfraRed Instrumentation Group, Oude Hoogeveensedijk 4, 7991 PD Dwingeloo, The Netherlands \\ d Department of Astrophysical Sciences, Princeton University, Princeton, NJ 08544, USA \\ e Leiden Observatory, Leiden University, PO Box 9513, 2300 RA Leiden, The Netherlands
}

\section{A R T I C L E I N F O}

\section{Article history:}

Received 9 June 2017

Accepted 28 February 2018

Available online 12 March 2018

\section{Keywords:}

Telescopes

Time-domain astrophysics

Astronomical databases

Surveys

Catalogues

Database query processing

\begin{abstract}
A B S T R A C T
Coming high-cadence wide-field optical telescopes will image hundreds of thousands of sources per minute. Besides inspecting the near real-time data streams for transient and variability events, the accumulated data archive is a wealthy laboratory for making complementary scientific discoveries.

The goal of this work is to optimise column-oriented database techniques to enable the construction of a full-source and light-curve database for large-scale surveys, that is accessible by the astronomical community.

We adopted LOFAR's Transients Pipeline as the baseline and modified it to enable the processing of optical images that have much higher source densities. The pipeline adds new source lists to the archive database, while cross-matching them with the known catalogued sources in order to build a full lightcurve archive. We investigated several techniques of indexing and partitioning the largest tables, allowing for faster positional source look-ups in the cross matching algorithms. We monitored all query run times in long-term pipeline runs where we processed a subset of IPHAS data that have image source density peaks over 170,000 per field of view $\left(500,000 \mathrm{deg}^{-2}\right)$.

Our analysis demonstrates that horizontal table partitions of declination widths of one-degree control the query run times. Usage of an index strategy where the partitions are densely sorted according to source declination yields another improvement. Most queries run in sublinear time and a few $(<20 \%)$ run in linear time, because of dependencies on input source-list and result-set size. We observed that for this logical database partitioning schema the limiting cadence the pipeline achieved with processing IPHAS data is $25 \mathrm{~s}$.
\end{abstract}

(C) 2018 Elsevier B.V. All rights reserved.

\section{Introduction}

High-cadence astronomy is a relatively new field in observational astronomy. Advances in hardware and software technology have made it possible to stream large volumes of observational data over fast links to clusters of computers that, in general, process the data in one or more automated pipelines for scientific analysis. The time available to do real-time analysis is limited by the cadence of the instrument. Therefore, additional and complementary scientific data analyses are forced to shift to non-real time environments. Here, all data accumulates over time and the growth may vary in the range of 0.1-100 PB/yr (Becla and Wang, 2014).

\footnotetext{
* Corresponding author.

E-mail address: bscheers@cwi.nl (B. Scheers).
}

These volumes clearly challenge many aspects of contemporary data management systems, which is also recognised by Ivezić et al. (2017).

Several instruments have shown impressive demonstrations of charting the sky down to a timescale of seconds, e.g., the international LOFAR telescope (van Haarlem et al., 2012), the Murchison Wide-field Array (MWA; Tingay et al., 2013), the Australian Square Kilometre Array Pathfinder (ASKAP; Murphy et al., 2013). High-cadence observations in image-domain astronomy, where sky regions are revisited many times in relatively short periods, produce overwhelmingly large amounts of data. Optical and radio telescopes planned for the next decade will generate even larger continuous data streams, e.g., the Large Synoptic Survey Telescope (LSST; Lazio et al., 2014; Juric and Tyson, 2015), the additional Ground-based Wide Angle optical Camera (GWAC; Cordier et al., 2015) of the Space-based multiband astronomical Variable 
Objects Monitor (SVOM), BlackGEM (Bloemen et al., 2015), the Square Kilometre Array (SKA; Broekema et al., 2012).

Although high-cadence instruments are specifically designed to carry out their own unique science, they share similar observational strategies. The main ones being: high-speed, wide- or all-sky surveys, searching for transient and variable sources on a variety of time scales and gradually archiving full-source lightcurve catalogues. In this respect, the archive is considered the new Big Data laboratory, equipped for making scientific discoveries in complex structured data. However, such discoveries are only possible when the infrastructure and software tools allow continuous and simultaneous data mining, statistical modelling, machine learning and ad-hoc querying.

The optical Sloan Digital Sky Survey (SDSS; York et al., 2000; Alam et al., 2015) was the first instrument to seriously integrate a database system into its survey design. It uses a database-centric computing approach for their large-scale scientific datasets. SDSS data are cumulatively released to the public in roughly annual cycles. In this respect, SDSS is a low-cadence instrument since the yearly updates of the full-source catalogue make the database essentially static.

On account of Gray's law to ship computations to the data instead of data to the computations (Szalay and Blakeley, 2009) many algorithms are designed to run inside the database engine. Another design rule includes knowledge of the 50 most frequent and intensive queries. Since astronomical pipelines process the data in a structured way, this allows one to optimise execution plans for known queries.

In the radio regime, the automated Transients Pipeline (TraP) of the international LOFAR telescope adopted many database techniques from SDSS (Swinbank et al., 2015). The TraP applies source finding and fitting to calibrated radio images after which, per image, all image and source properties, i.e. the source list, are handed over to the database. Note that the images themselves are not stored in the database. The loop of tasks of the TraP database consists in total of about 50 queries which can be divided into four successive steps, all executed in bulk mode:

1. load source list

2. cross-match source list with catalogue of known sources

3. update catalogue: maintain up-to-date statistical sky model

4. find/identify transient and variable sources or other significant deviations from the sky model

Typical source lists for LOFAR survey-mode observations do not exceed $10^{3}$ entries, whereas averages are less than $10^{2}$ for cadence modes as high as $10 \mathrm{~s}$ (Swinbank et al., 2015). The total number of unique sources in the LOFAR radio catalogue is of the order $10^{6}$. Long-term monitoring of the database tasks is essential to predict pipeline performance and understand the instrument as a whole. Queries with poor scaling (e.g. exponential) will eventually jam the processing. Significant increases of cadence and/or source density determine the critical limits of the system and permitted types of observations. Swinbank et al. (2015) show that the TraP run times increase linearly with input size within the LOFAR observation constraints.

Source lists produced by optical instruments are in general much larger, primarily due to the intrinsic higher resolution in combination with the increased sensitivity. Also the catalogues that represent the optical sky models hold orders of magnitude more sources than their radio counterparts. Therefore, one avoids naive implementations of the TraP for optical instruments, since the extrapolation of the source counts into the optical spectrum will most probably break linear performance or even in a best-case long-term linear performance scenario, the processing time will pass the cadence time at some point.
Table 1

Characteristics of the MeerLICHT telescope, a single BlackGEM prototype telescope. DB source data size is the storage size that all properties of single source would need when stored in a database. We assume observation nights of $10 \mathrm{~h}$.

\begin{tabular}{|c|c|c|}
\hline Mirror diameter & \multicolumn{2}{|c|}{$65 \mathrm{~cm}$} \\
\hline FoV & \multicolumn{2}{|c|}{$2.7 \mathrm{deg}^{2}$} \\
\hline CCD size & \multicolumn{2}{|c|}{$10,536 \times 10,536$} \\
\hline Resolution & \multicolumn{2}{|c|}{$0.57^{\prime \prime} / \mathrm{px}$} \\
\hline bits per px & \multicolumn{2}{|c|}{16} \\
\hline Image size & \multicolumn{2}{|c|}{$222 \mathrm{MB}$} \\
\hline Calib. images per night & \multicolumn{2}{|c|}{$2 \times(10$ bias $+5 \times 5$ flats $)=70$} \\
\hline DB source data size & \multicolumn{2}{|c|}{$402 \mathrm{~B}$} \\
\hline Observation mode & Nominal & Fast \\
\hline Integration time & $5 \mathrm{~min}$ & $1 \mathrm{~min}$ \\
\hline Sensitivity & $23 \mathrm{mag}$ & $21 \mathrm{mag}$ \\
\hline Science images per night & 120 & 600 \\
\hline Data rate per night & $42 \mathrm{~GB}$ & $148 \mathrm{~GB}$ \\
\hline
\end{tabular}

The planned wide-field optical telescope array BlackGEM is dedicated to measure optical emission from pairs of merging neutron stars and black holes (Bloemen et al., 2015). BlackGEM will start with 3 telescopes, all of which will be located at ESO La Silla, Chile. MeerLICHT, ${ }^{1}$ a single BlackGEM telescope acting as a prototype, is coupled to the MeerKAT radio array (a precursor to SKA; Brederode et al., 2016) to operate in concert and allowing to study the optical-radio sky simultaneously as a true multiwavelength instrument. Table 1 shows the characteristics of a single BlackGEM telescope. From a database-pipeline perspective, the most influential properties are the source data size, the source density and the integration time, where the latter determines the cadence.

In this paper we use the TraP-like queries as a baseline and investigate its scalability to the MeerLICHT environment. We need to know to what extent the processing of images with source densities of $500,000 \mathrm{deg}^{-2}$ or source lists with hundreds of thousands of sources is still feasible.

The paper is outlined as follows. Section 2 gives the rationale behind the choice of a column-oriented relational database management system (RDBMS). Section 3 describes the experimental set up of the performance tests, the data and the TraP queries that were adjusted and optimised. The results are presented in Section 4 and concluded in Section 5. Although all source codes are publicly available, the Appendices A-C show the relevant query snippets for readability.

\section{Rationale for MonetDB, a column-oriented relational database management system}

Non-relational databases, e.g., key-value and NoSQL stores, lack solid support for many data manipulations that are required for this type of astronomical application. We cannot afford redundant or duplicate storage, and therefore have to distribute the data over a minimal set of related tables. The absence of fast join and crossmatch functionality, schema free storage formats, no transactional support and own unique query languages makes non-relational databases hard to perform on such applications. Furthermore, the bulk processing requires fast data aggregation, ordering and indexing to avoid large table scans. All these functionalities are wellknown and implemented in relational database systems.

Relational database storage models follow either the roworiented or column-oriented principles. The row-oriented storage model partitions tabular data horizontally. Such record layouts consist of rows that store all their columns contiguously, with the adverse effect that a data block contains multiple data types.

1 Check the current status at www.meerlicht.org. 
Queries that touch only a few columns of a large table or joins of tables, waste both bandwidth and memory space in this model, because the blocks that the CPU reads and buffers are contaminated with all the other, unwanted, columns. Abadi et al. (2008) discuss more differences between row and column stores.

The columnar model splits tabular data vertically. Every column is represented by an array of single data type. These kinds of array can be manipulated easily and scanned quickly when sorted. Its variables of fixed size are densely stored, possibly in compressed format, on disk. The uniformity of the array fits well to the blockoriented nature of memory transfers and CPU caches and exposes good spatial locality and high cache hit ratios when queries execute large scans over a subset of columns. More in-depth details of column-oriented databases can be found in Abadi et al. (2012).

More research and developments in the field of columnoriented databases led to the implementation of many related techniques in the open-source main-memory relational database management system MonetDB (Boncz, 2002). MonetDB's architecture is geared toward read-optimised data-intensive scientific applications (Zukowski, 2005). It is compliant with the SQL2008 standard and has language bindings for C, Java, Python, $R$ and JavaScript/Node.js. The ease of extending its functionality with user-defined functions (UDFs) written in SQL, C, R and Python are other serious strengths. MonetDB follows a strict columnar design and takes into account the underlying computer architecture (Boncz et al., 1999; Héman, 2015). The fundamental removal of the expression interpreter fully eliminates parsing expensive code, verifying record layouts and checking data types. Hard-coded semantics makes MonetDB's algebra simple yet efficient, because all operators work on simple arrays allowing the compiler to generate CPU-friendly instructions. Column-at-atime processing reduces the number of function calls and data and control dependencies, which in turn improve the algorithms and the CPU cache performance, as opposed to tuple-at-a-time iterators. Look-up and range queries benefit from hash-indexes and secondary imprints indexes, resp., that are automatically built for touched columns (Sidirourgos and Kersten, 2013). A query optimiser generates and analyses alternative query plans and executes the plan that minimises the query cost. Manegold et al. (2002) developed cost models that estimate the query execution time based on I/O and average CPU costs. Ivanova et al. (2013) extended MonetDB's code base to support SQL management of external data (SQL/MED). Loading binary columnar catalogue FITS files in this way is orders of magnitude faster than using classical statements, since the in-memory binary data exactly matches MonetDB's storage model.

Until now, the largest database archive for a single telescope is the SDSS SkyServer tuned to the commercial closed-source row store Microsoft SQL Server. Full-sized data releases were successfully ported into MonetDB. Ivanova et al. (2007) demonstrated that MonetDB is capable of loading and querying the SDSS SkyServer data. Initial performance evaluations on a smaller subset indicated that $85 \%$ of the most executed queries run faster in MonetDB, while the remaining are of competitive speed. Most of the time scientific queries only touch a few columns, whereas the tables generally have many hundreds of columns. This is a strong call for using column-oriented databases

In all our experiments we use the Structured Query Language (SQL) to interact with the data. SQL queries access data directly and return aggregated or full result sets, in contrast to scripts that retrieve datasets and process tuples iteratively. (Note that in this context a query is a generic term for any kind of instruction set(s) that run on database data.) Query response times are critical in high-cadence pipeline applications. Because it is more efficient to process data in bulk mode, the fact that data access is fastest close to the CPU and that astronomical queries very often work on columns or ranges thereof, the choice for a main-memory columnoriented database in astronomical pipelines is obvious.

During database kernel and pipeline query development we ran comparison tests with main alternative open source RDBMSs regularly. However, reports of these results are beyond the scope of this paper.

\section{Baseline and alternative high-cadence pipeline benchmarks}

\subsection{BlackGEM pipeline architectures}

BlackGEM will produce about $1 \mathrm{~TB}$ of data per night of which 90\% are raw and calibrated images and 10\% extracted information for the full-source database. Raw data are calibrated and imaged after which the source-extraction output product is a list of all detected sources and their properties in binary catalogue FITS format.

A primary image-differencing pipeline runs in real time to detect transient and variable events in the data stream of calibrated images. For differencing it uses reference images and parts of the source list for PSF fitting. The output product is a binary catalogue FITS file of all transient sources, which will be stored in a separate relatively small transient-source database, which will not be discussed any further here.

The full-source binary catalogue FITS files serve as input for a secondary pipeline that runs in an offline mode and consistently stores all sources into the full-source database for scientific analysis. Delays in this mode are acceptable up to the point where the overall cadence is not being met anymore. This paper concentrates on the secondary pipeline and its full-source database. The high cadence and source densities of BlackGEM and MeerLICHT force us to carefully monitor the long-term run-time performance of the pipeline queries, especially since they run in a dynamically growing database.

The pipeline processes the data in a number of steps described in Section 1. After the sources have been loaded into the database (step 1), read, write and delete queries take care of the source association and sky model maintenance procedures (steps 2 and 3 ).

It needs to be noted here that transactions in MonetDB follow the optimistic concurrency control scheme. The lack of a locking scheme implies that queries modifying the data (of which we have many) are serialised at the application level and run in singlethreaded mode. Explicitly programmed multiple threads with own database connections can run in parallel easily if the query's write operations are executed on independent tables, however, we did not code that in our modules. On the other hand, queries that only read data run in parallel multi-threaded mode implicitly.

\subsection{IPHAS data}

Before BlackGEM is operational we process real binary catalogue FITS files from the Isaac Newton Telescope (INT) Photometric $\mathrm{H} \alpha$ Survey of the Northern Galactic Plane (IPHAS; Barentsen et al., 2014). The $10 \sigma$ limit is at magnitude 20 and the field of view is $0.29 \mathrm{deg}^{2}$. IPHAS has about 200 observations per night, where single source lists range between 1000 and 130,000 entries, with peaks up to 170,000 .

\subsection{The SciLens cluster}

We run our experimentation queries on multiple nodes of the SciLens $^{2,3}$ cluster located at CWI. Our queries ran on single cluster nodes, to which the input files were transferred. Table 2 gives an excerpt of the specifications of the nodes that we used.

\footnotetext{
2 http://www.scilens.org.

3 For the current status and overview of the configuration, see https://www. monetdb.org/wiki/Scilens-configuration-standard.
} 
Table 2

Configuration of SciLens cluster nodes.

\begin{tabular}{|c|c|c|c|c|c|}
\hline & & diamonds & stones & bricks & rocks \\
\hline \multirow[t]{6}{*}{ CPU } & \multirow{6}{*}{$\begin{array}{l}\text { Architecture } \\
\text { CPU(s) } \\
\text { Threads per core } \\
\text { Cores per socket } \\
\text { Socket(s) } \\
\text { Clockspeed }\end{array}$} & \multicolumn{4}{|c|}{$\mathrm{x} 86 \_64$} \\
\hline & & 96 & \multicolumn{2}{|c|}{32} & 8 \\
\hline & & \multicolumn{4}{|c|}{2} \\
\hline & & 12 & \multicolumn{2}{|c|}{8} & 4 \\
\hline & & 4 & \multicolumn{2}{|c|}{2} & 1 \\
\hline & & $2.4-2.9 \mathrm{GHz}$ & $2.6-3.4 \mathrm{GHz}$ & $2.0-2.8 \mathrm{GHz}$ & $3.4-3.8 \mathrm{GHz}$ \\
\hline RAM & Size & 1024 GB & \multicolumn{2}{|c|}{$256 \mathrm{~GB}$} & $16 \mathrm{~GB}$ \\
\hline \multirow[t]{2}{*}{ SSD } & \multirow{2}{*}{$\begin{array}{l}\text { Drives } \\
\text { /ssd }\end{array}$} & & & $8 \times 128 \mathrm{~GB}$ & \\
\hline & & & & 8x HW RAID0 & \\
\hline \multirow[t]{3}{*}{ HDD } & \multirow{3}{*}{$\begin{array}{l}\text { Disks } \\
\text { /scratch }\end{array}$} & $4 \times 2 \mathrm{~TB}$ & $3 \times 3 \mathrm{~TB}$ & $4 \times 2 \mathrm{~TB}$ & \multirow{3}{*}{$\frac{1 \times 2 \mathrm{~TB}}{1.8 \mathrm{~TB}}$} \\
\hline & & $\begin{array}{c}7.2 \mathrm{~TB} \\
(4 \times \text { HW RAID0 })\end{array}$ & $\begin{array}{r}5 \\
(3 \times \mathrm{SV} \\
\end{array}$ & $\begin{array}{l}\text { TB } \\
\text { RAID0) }\end{array}$ & \\
\hline & & & $3 \times 0.9 \mathrm{~TB}$ & $1.8 \mathrm{~TB}$ & \\
\hline \multirow[t]{2}{*}{ Network } & \multirow{2}{*}{$\begin{array}{l}\text { Ethernet } \\
\text { Infiniband }\end{array}$} & $2 \times 10 \mathrm{~Gb} / \mathrm{s}$ & \multicolumn{3}{|c|}{$1 \mathrm{~Gb} / \mathrm{s}$} \\
\hline & & $4 \times 40 \mathrm{~Gb} / \mathrm{s}$ & \multicolumn{3}{|c|}{$40 \mathrm{~Gb} / \mathrm{s}$} \\
\hline \multirow[t]{3}{*}{ Software } & \multirow{3}{*}{$\begin{array}{l}\text { OS } \\
\text { MonetDB } \\
\text { Python }\end{array}$} & \multicolumn{4}{|c|}{ Linux, Fedora 24 4.7.3-200.fc24 } \\
\hline & & \multicolumn{4}{|c|}{ Jun2016 SP1 } \\
\hline & & \multicolumn{4}{|c|}{2.7 .12} \\
\hline
\end{tabular}

\subsection{Baseline pipeline and query monitoring}

We used the May 2007 binary catalogue FITS files as input for the prototype pipeline. The series consists of 1893 files, where each FITS file has four extensions due to the four CCDs of the telescope. The Python prototype pipeline script is based on the TraP (Swinbank et al., 2015) and was modified for the load and cross-match stages (steps 1 and 2) before it could serve as the baseline pipeline.

The most significant change in the data loading of step 1 was the replacement of the classical SQL insert statements with SQL/MED queries (Ivanova et al., 2013). This technique attaches datasets to the database that can be queried before they are actually loaded. Larger files, i.e., longer source lists, can be handled, because the data do not need to be parsed. In step 2, the variable conical search radius that is being used for cross-matching radio sources was set as a constant parameter because of the more stable quality of optical images (see Section 3.5).

All individual query run times are written to log files for easy plotting and identification of $\mathrm{CPU}$ and $\mathrm{I} / \mathrm{O}$ intensive queries. Sets of queries that belong to a step as described in Sections 1 and 3.1 are grouped together and prefixed I or A for loading/inserting or cross-matching/associating, resp. The modified TraP, as described in the previous paragraph, is considered as the baseline pipeline to which the query optimisations will be compared.

All source codes presented in this paper is publicly and freely available for download and usage at the CWI git-repository site. ${ }^{4}$ It also provides instructions to get started.

\subsection{Cross matching and baseline module MO}

We adopted the set of TraP source association queries, including the cross-match query, and modified it slightly for the processing of optical data. This then is our baseline module MO. The source association queries are the most intense and sensitive database operations, where a source list is cross-matched with the internal catalogue of known sources. At the core of this query is the positional source look-up by a conical search. From SDSS SkyServer log analyses Ivanova et al. (2007) found that these conical searches were part of the most frequently executed queries. Optimising this will minimise CPU time and compensate processing times for other queries.

\footnotetext{
4 https://scm.cwi.nl/DA/blackgem-code. The git version used for this paper was 3ad947789a19.
}

The cross-matching method applied here is based on the TraP source association described in Section 4 of Swinbank et al. (2015). In that paper it was also shown that the association algorithms scale reasonably linear with the number of sources. Source lists in their performance tests, however, do not exceed 1200 entries, which is acceptable in the radio domain of LOFAR, but not in the optical regimes of MeerLICHT and BlackGEM. The TraP is untested for source densities that are orders of magnitude larger, making it plausible that we cannot simply extrapolate the TraP test results to the optical domain.

The resolution and positional uncertainties in the optical IPHAS images are more stable compared to the LOFAR case, where the image resolutions fluctuate due to more complex radio-specific calibrations and antenna dependencies. Therefore, the TraP implements a variable search radius to cross-match sources, but because the optical image quality is more stable we can replace it in the query algorithm by a simpler conical search radius of constant value that is determined by the telescope's resolution element.

The cross-match baseline query is written out in Appendix B. It joins the sources from the latest appended source list available in the extractedsource table with the known catalogued sources stored in runningcatalog. If the distance between a found source pair is less than the search radius the pair is considered as a genuine association and both IDs and distance are returned. In fact, however, more properties are returned, but for illustrative purposes they are omitted here. It must be noted here that the crossmatch query result set does not contain only unique catalogue source-extracted source pairs. Multiple types of association pairs are possible, falling into the categories of no association, one-toone, many-to-one, one-to-many and many-to-many. The association module takes care of further processing these topologies, which are described in detail by Swinbank et al. (2015).

The on-sky distance is only calculated for catalogue counterparts that lie within the boxes centred at the source positions from the list. The widths of the boxes are determined by the fixed search radius. Both tables have an integer zone column of 8-bit data type, that specifies the declination strip in which the source lies. The box height gives which neighbouring declination strips need to be searched. The on-sky search radius is constant, but expressed in RA it varies depending on declination. Therefore, the RA-box width increases when moving towards the celestial poles. The user-defined alpha () function determines the rate of inflation as given by Gray et al. (2006).

The distance in radians on the sky, $\vartheta$, between a measured source position, $\mathbf{x}$, and its candidate counterpart in the running 
catalogue, $\mathbf{m}$, is given by the dot product $\mathbf{x}^{T} \mathbf{m}=\cos \vartheta$, where $\mathbf{x}$ and $\mathbf{m}$ are unit vectors. However, when dealing with small angles the alternative of using the sine function gives computationally more accurate results. Therefore we use the arc-angle distance between $\mathbf{x}$ and $\mathbf{m}$ to determine $\vartheta$

$\sin \frac{1}{2} \vartheta=\frac{1}{2}\|\mathbf{x}-\mathbf{m}\|$.

The above mentioned box size and distance criteria are declared in the cross-match baseline SQL statement (see Appendix B). Naive SQL execution plans in the case of large source lists may downgrade the performance to unacceptable levels. Therefore, we have to carefully design several alternative cross-match queries that implement different search techniques and corresponding query optimisations in order to evaluate their performance under different circumstances.

In the next paragraph we will elaborate on alternative queries, in a database schema where we partition and sort the largest tables by declination.

\subsubsection{Alternative module $M 5$}

Data partitioning is a well-known concept in database designs to control load balancing and performance. Tables are divided into independent smaller (sub)tables, which can be accessed at a finer level of granularity. This functionality allows a table to be defined as a union of its partitions. One then can query the parent table as if it is an autonomous table..$^{5}$ On the other hand, for finer control and better data locality one can query individual partitions, which are tables, to avoid access to partitions that are outside the query. In module M5 we investigate the performance of the cross-matching pipeline where the largest tables are partitioned horizontally into declination zones of one degree.

We force the partition tables to be sorted according to declination, which uses the system cores in parallel. This means that when table rows need to be updated or appended, the partition table is rewritten. This is acceptable as most of the table chunks are sorted according to their original format. Moreover, cross-matching will be fast and can be done in memory, since the tables are relatively small and in sorted order. The partitioning scheme is also prepared to withstand database growth, since the partition sizes do not exceed the expected number of sources. The relevant M5 SQL code is shown in Appendix C.

\section{Presentation of experimental results}

The baseline (M0, Section 3.5) and alternative (M5, Section 3.5.1) modules both have the same set of SQL/MED loading queries, but a different set of cross-matching queries. On the same type of node the same loading queries compete for memory and CPU with different sets of cross-matching queries from the respective modules. Every cross-matching module has its specific methods of memory allocation and data storage, which produce unequal loading behaviour. Nevertheless, the variations between modules are small and therefore Section 4.1 only presents the loading query run times of the alternative module M5. In Section 4.2 we present the details of different cross-matching modules and in Section 4.3 the overall performances.

\subsection{Loading part}

The loading part consists of nine queries that first attach and load the binary FITS files into temporary tables that are created on the fly (I1-I3), then copy the FITS header data and sources over into permanent tables (I4 and I5, resp.) and finally clean up

\footnotetext{
5 More on the internals can be found at https://www.monetdb.org/ Documentation/Cookbooks/SQLrecipes/DataPartitioning.
}

the schema (I6-I9) before the next file comes in. The accumulated run-time performances of the individual queries and the growth of the number of sources in the database with respect to the FITS image sequence for the alternative module M5 on the diamonds node are shown in the left graph of Fig. 1. The thick (red) line in this graph represents the number of sources in the database at the moment of query execution and corresponds to the number of entries in the largest table, which is a measure for the database size. Although the number of sources increases irregularly, the individual query run times accumulate linearly over time, meaning a query runs equally fast at any moment.

The constant-time complexity $\mathcal{O}(1)$ is clearly visible in the right graph of Fig. 1, where the individual query run times are plotted vs. the number of sources in the database. Although there is some scatter on the nodes that have limited memory in combination with the slower HDDs (not plotted here), these queries run independent of the database size. It is not possible to make a fair comparison with the loading query set of the TraP as presented by Swinbank et al. (2015), because they do not separate the loading from the cross-matching in their performance plots. However, the flat performance of the SQL/MED queries for the baseline and alternative modules allows the loading of source lists that more than 100 times larger.

Queries I3, I4 and I5 (spelled out in Appendix A.1, Appendix A.2 and Appendix A.3, resp.) contribute most to the total load time. Query I3 is an SQL/MED procedure call. After the FITS data have been attached to the database by generating a fully queryable temporary table, this call really loads the data into the database, effectively making the data-vaults table permanent in the database schema. Query I4 loads the header data, common to all sources originating from the same FITS file, into the image table as a single entry, whereas I5 appends all data from the datavaults table to the permanent extractedsource table. Such bulk inserts vary, but can be over $10^{5}$ entries (see Section 3.2). The insertion of only one entry into the image table is an expensive operation, because all fifteen data types per entry have to be parsed. Comparison to the other append/insert queries (I1, I3 and I5) makes the effect more conspicuous, where the cost of appending $10^{5}$ sources is low since the SQL queries are aware of attaching binary data and the data types. However, all load queries run in constant time, independent of the database size with the ability to scale up even further.

\subsection{Cross-matching part}

After the sources from a FITS file have been inserted the crossmatching procedure starts and runs 15 to 46 queries depending whether the baseline or alternative module was chosen. The source list is cross-matched with counterparts in the catalogue of known objects. As described in Section 3.5 this results in a candidate list that is further sifted by subsequent queries to resolve the various association types of source-object pairs that turn up. Swinbank et al. (2015) discuss the different types and elaborate on the devised steps, i.e., queries, to append the new source measurements to existing or new light curves. The final step is to update the statistical properties of the known catalogue sources to include the new measurements in the model. Sources for which no counterparts were found are appended as new entries in the catalogue.

There are many queries involved in this module, but we will focus on the query that performs the cross-matching.

\subsubsection{The baseline cross-match module MO}

The baseline cross-match module consists of fifteen queries in total of which the cross-matching query is labelled Q11b (see Appendix B). The graphs in Fig. 2 show the performance of the cross-matching module as a whole (left column), the other queries 

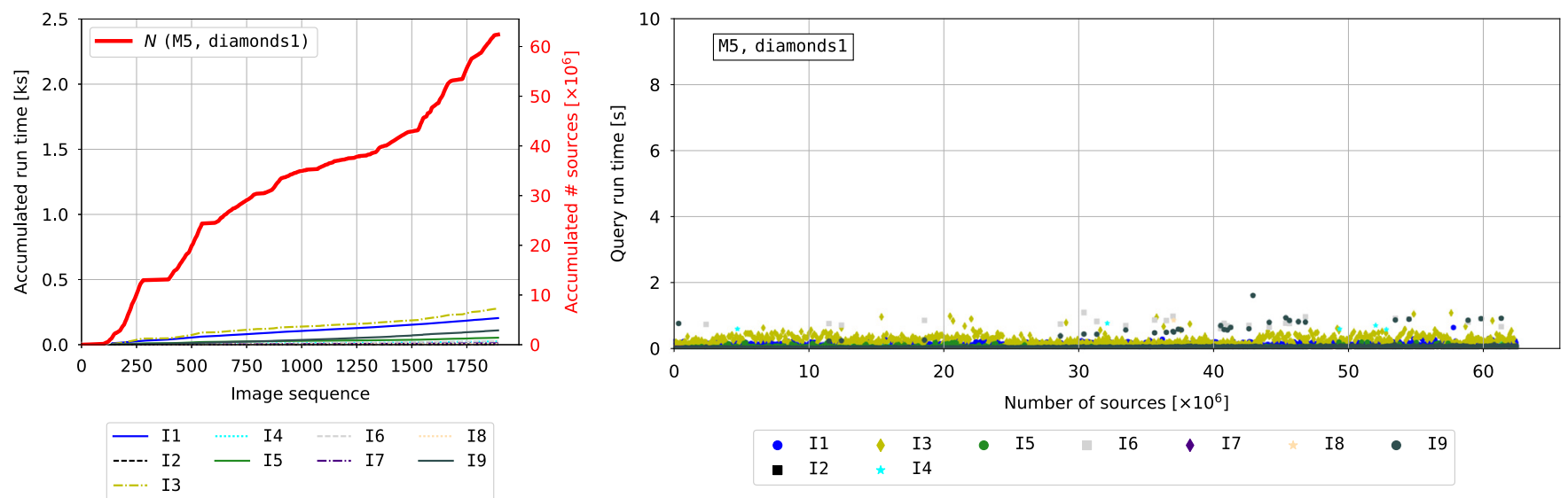

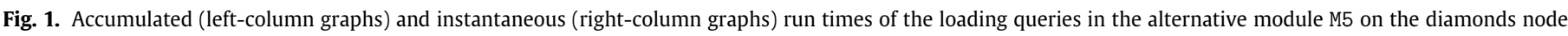

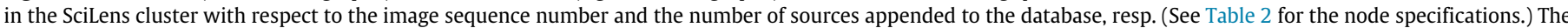

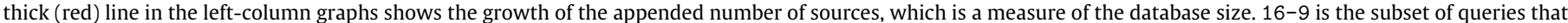

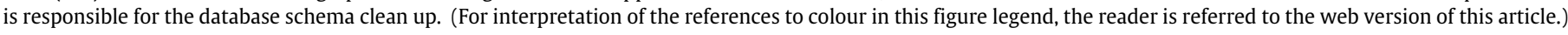

(middle column) and the individual cross-matching query Q11b (right column), while the rows specify the types of node on which the pipeline ran.

The left-column graphs show the run times summed over all fifteen queries vs. the image sequence number. During a pipeline run the total run time to process a single FITS image fluctuates heavily and depends strongly on the growth rate of the database size. Larger data input sizes will slow down the pipeline run.

From the right-column graphs it can be seen that the crossmatching query run times are of the same order as the above mentioned summed query run times, meaning that the pipeline run time is determined by $\mathrm{Q} 11 \mathrm{~b}$. We have divided the crossmatching query run times in these graphs into three consecutive parts according to their image sequence number: the first 500 images, the middle part of images 500-1500 and the remaining images starting from sequence number 1500 . This points out that cross matching is not only a function of number of query rows, but also of database size, since for the same number of rows the query run times slow down as the database fills up with sources. Searching for counterpart candidates takes longer, since larger tables have to be scanned.

Comparisons of the run times of the cross-matching query to the other queries, shown in the graphs in the middle column of Fig. 2, reveal a difference of more than two orders of magnitude, making the cross-matching query the most dominant one in module MO. The middle graphs show that most queries evolve linearly in time as the database size grows, while only a few run in constant time independent of the database size.

It all indicates that the cross-matching query and thus the baseline module scales with database size in $\mathcal{O}(N M)$ time, where $N$ is the number of sources in the database and $M$ the number of sources in the resulting candidate list, which is approximately equal to the number of entries in the source list originating from the FITS file.

The pipeline performance on the diamonds and bricks nodes is similar, because for the former the RAM size is large enough and for the latter RAM size is sufficient in combination with the low latency storage access of the SSDs. On the stones and bricks nodes some of the other queries are memory bound. The fast CPU of the rocks node does not compensate its small RAM size, which effect is more prominent when the database size is larger. In these cases the operating system starts memory swapping data from RAM to disk, a process that impacts the pipeline performance negatively. The larger storage access latency for HDDs as compared to SSDs makes the pipeline runs slower and less smooth on a stones node than on a bricks node, despite its faster CPU.

\subsubsection{The partitioned cross-matching module $M 5$}

In module M5 the catalogued sources are distributed over multiple tables according to their declination zone as described in Section 3.5.1. The number of rows a query touches is now limited by the size of the partitioned tables, which is for zone widths of 1 degree two orders of magnitude smaller than the unpartitioned version. Query run times now only depend either on the number of rows of partitioned tables or on the size of the result sets instead of the size of the entire database. This is nicely demonstrated in Figs. 3 and 5, where the run-time performance of all queries in module M5 is shown.

The cross-matching query, labelled Q32 in module M5, is shown in the right-column graphs of Fig. 3. Because the partitioning restricts the number of sources, the query run times do not depend on the database size anymore. Therefore, in contrast with the baseline module, the cross-matching run times do not hinge on the image sequence number, but are determined by the number of entries in the result set, i.e. the number of counterpart candidates, which is approximately equal to the number of entries in the source list. This makes the M5 cross-matching query to run stable and faster over time than its counterpart in module MO.

The summed query run times fluctuate irregularly over the course of images, as can be seen in the left column of Fig. 3. This originates from query contributions that depend mainly on the input source-list size, which varies from image to image, but the run times never exceed the cadence time. The middle graphs in Fig. 3 show all queries that run in constant time. It can be seen that for the different nodes the scatter of the run times increases with decreasing RAM size. This is caused by queries that run in linear-time complexity and compete with all queries for the same memory resources. Queries that depend on the number of rows need to allocate a relatively larger percentage of total memory on the nodes with smaller RAM sizes, leaving a smaller amount to the remaining queries which in turn results in longer query run times.

The small group of queries that scales linearly with the number of rows are displayed in Figs. 4 and 5. Fig. 4 shows two queries of which the run time increases linearly with respect to the database size. The respective queries select the maximum ID value of the growing tables of extracted (Q12) and catalogued sources (Q19). Note that the latter is a MERGE table of many partitions. Since the number of unique sources is smaller than the total number of extracted sources the slope of query Q19 is less steep. Although it is not noticeable in these experiments, but if we extrapolate the database size to larger numbers, the run times will hit the cadence time at some point. A fairly easy way to correct this part to run in 

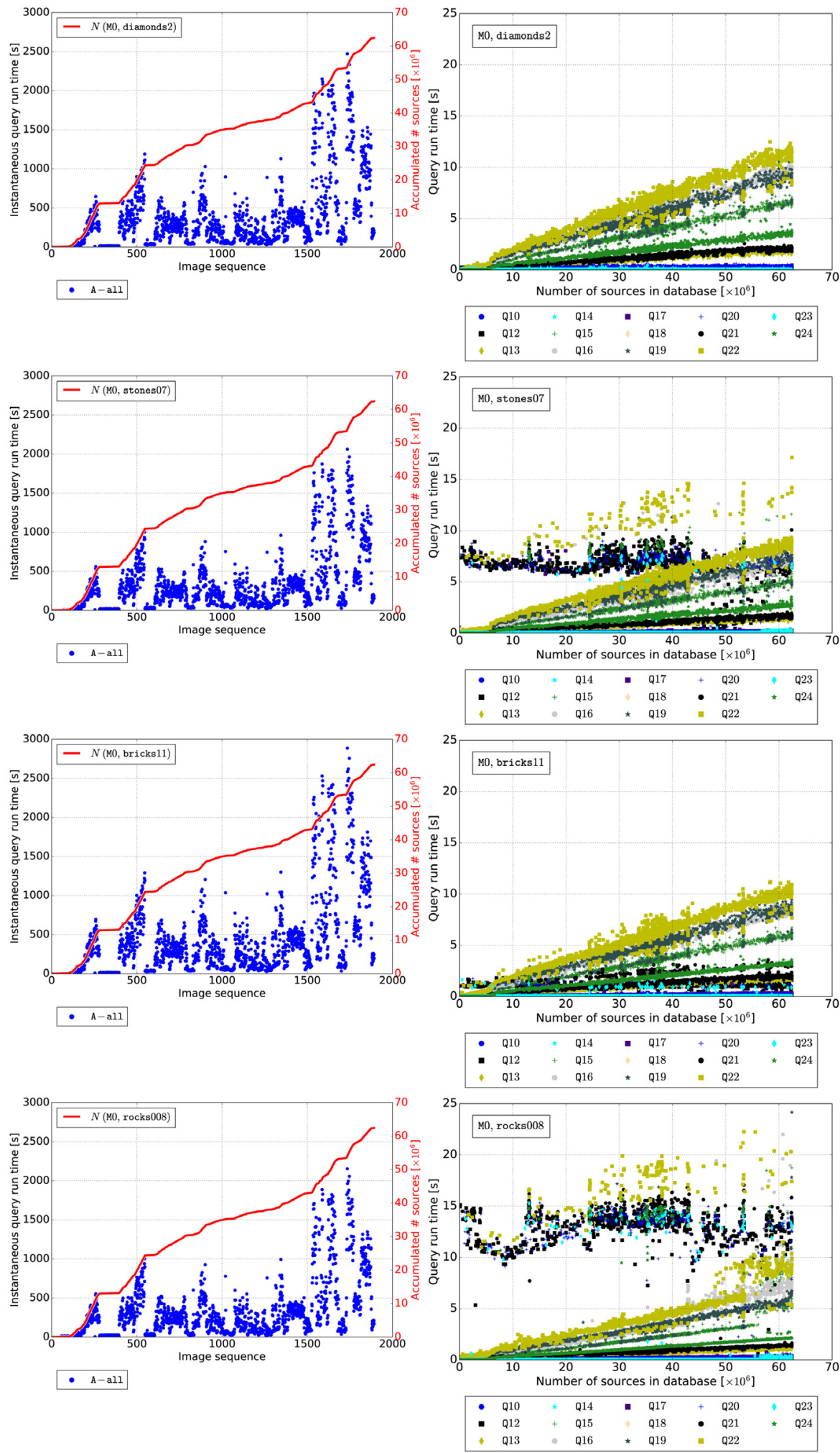
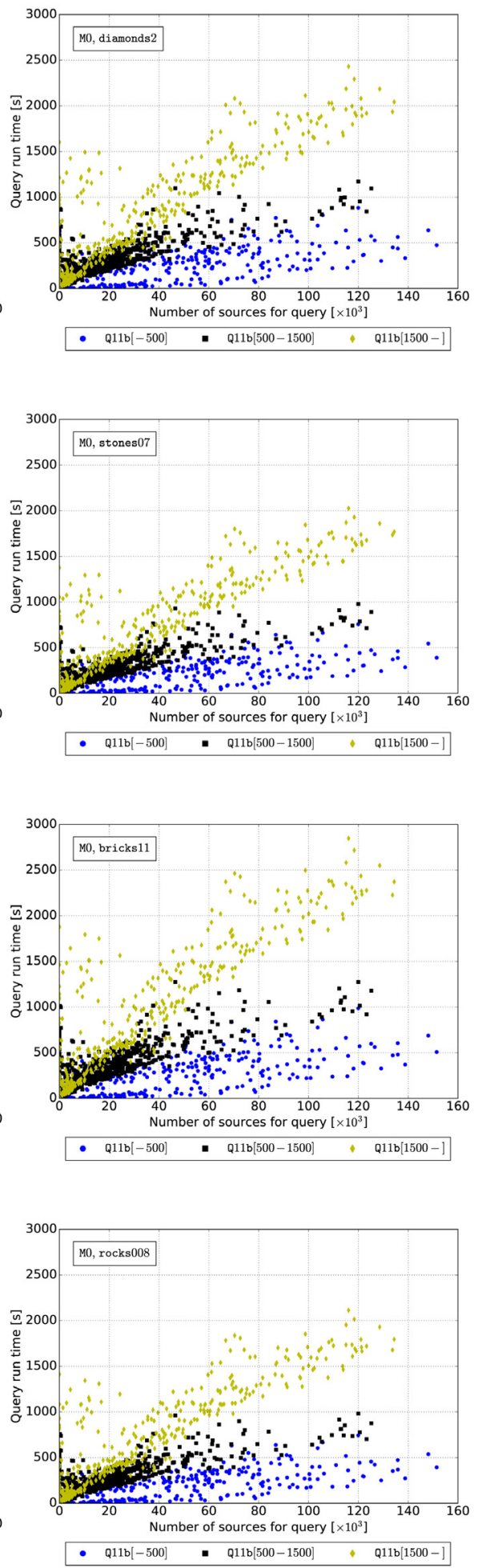

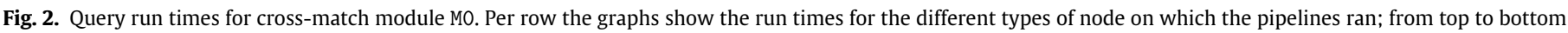

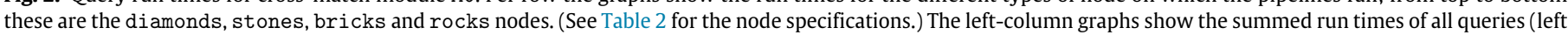

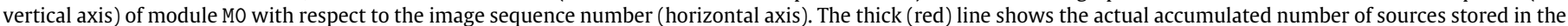

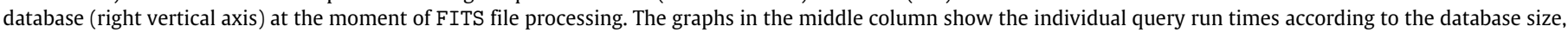

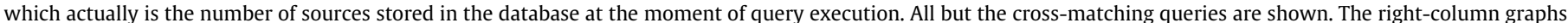

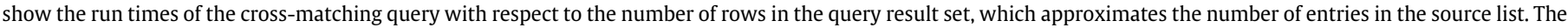
run times are divided into three parts, where the image sequences run from start to 500,500 to 1500 , and from 1500 up to the end. 

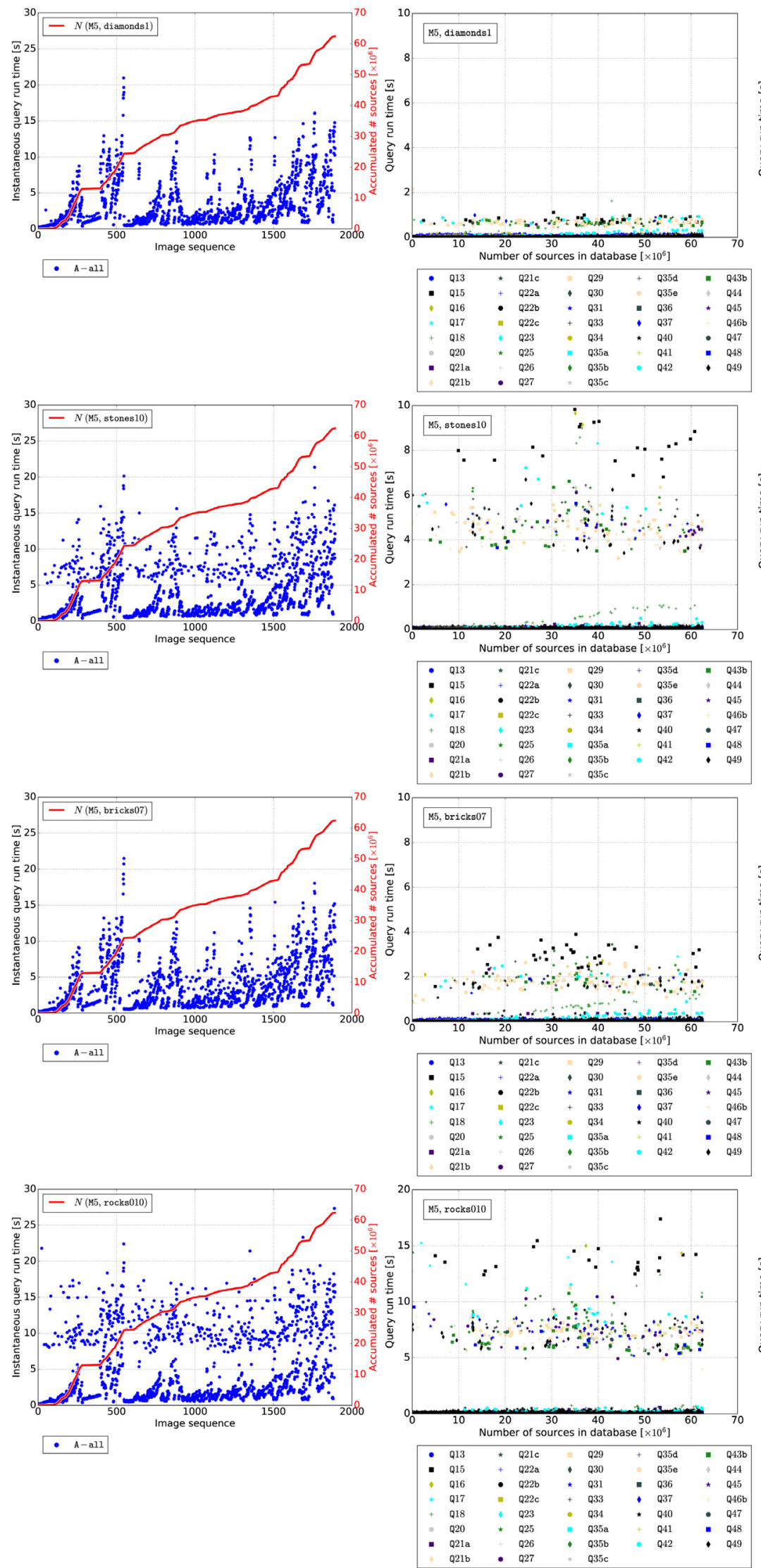

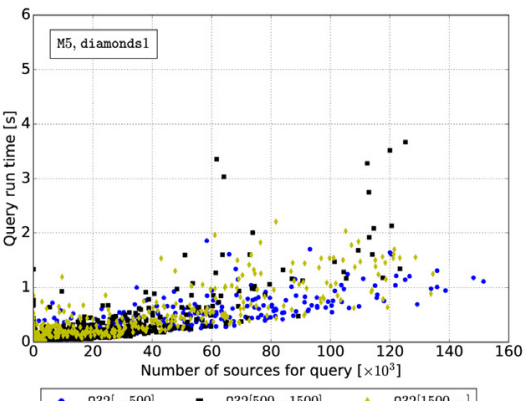

- $\mathrm{Q} 32[-500]$ - $\mathrm{Q} 32[500-1500] \& \mathrm{Q} 32[1500-]$

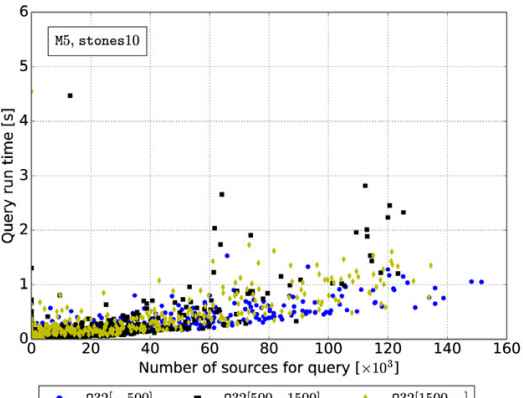

- $\mathrm{Q} 32[-500]$ Q $\mathrm{Q} 32[500-1500]+\mathrm{Q} 32[1500-1$
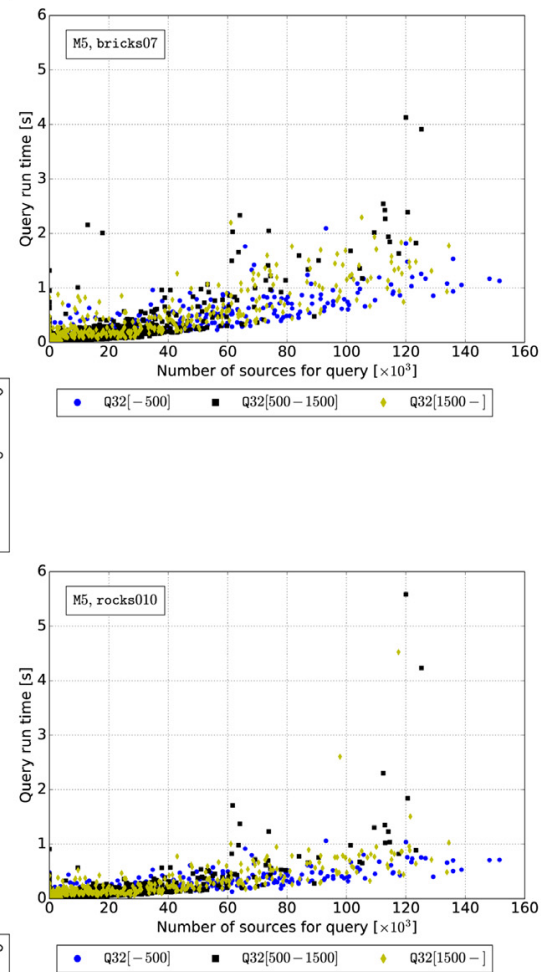

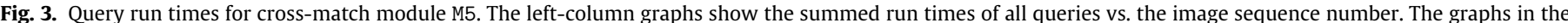

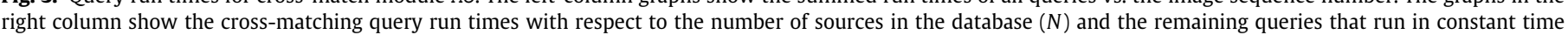

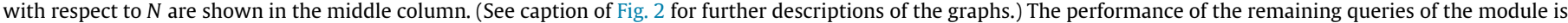
depicted in Figs. 4 and 5. 


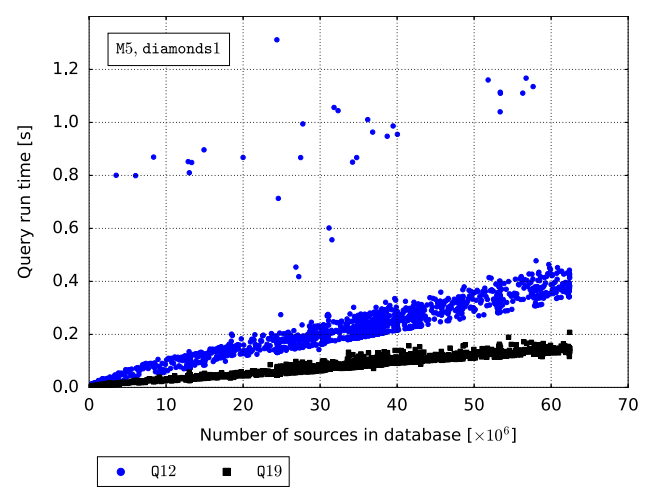

Fig. 4. Run time vs. number of sources in the database of the two queries that call the SQL MAX function in module M5 on the diamonds node.

Table 3

Accumulated processing times (in ks) of the modules for a complete pipeline run specified per type of node. Node rows give the specific node on which the pipeline ran, pipeline rows specify the total pipeline run time and query rows report the total run times of all queries summed.

\begin{tabular}{lllcrr}
\hline Module & & Diamonds & Stones & Bricks & Rocks \\
\hline M0 & Pipeline & 659.0 & 575.1 & 750.6 & 589.4 \\
& Query & 658.4 & 572.7 & 749.8 & 585.6 \\
\hline \multirow{2}{*}{ M5 } & Pipeline & 9.6 & 14.8 & 12.0 & 23.2 \\
& Query & 6.5 & 7.8 & 7.0 & 10.0 \\
\hline
\end{tabular}

constant time would be to maintain the values at the application level. However, a solution where the database itself keeps track of statistical parameters, e.g., average, minimum, maximum, standard deviation, is more elegant and is considered in future releases.

The graphs of Fig. 5 show the M5 query run times that do not run in constant time. The areas of the query run times are confined to certain regions. Queries Q46a and Q28 are working on the light-curve table and take into account an order of magnitude more entries than the queries that run over the catalogue table with unique sources (Q43a and Q24, where the latter is hidden behind the markers of other query run times). All four query run times depend on the number of sources in the partitions, i.e., the source density. Numbers of new datapoints appended to the lightcurve table (Q39) do not exceed $10^{6}$, whereas new entries for the extracted source table (Q14) and catalogue table (Q38) both do not exceed the number of source list entries. The graphs show that the performance is controlled and that the queries run at acceptable speeds.

\subsection{Overall pipeline runtime}

Table 3 reports the total time of the pipeline runs and the summed execution time of all queries, i.e. loading and crossmatching, for the baseline and the alternative module, specified per cluster node. It shows that absolute increment of the pipeline overhead is more prominent in the alternative module M5 than in the baseline module MO. This is caused by fact that we do not include the query commit times in the query runtime results. The larger number of queries and thus commits in the alternative module contribute more to the total pipeline runtimes.

The scalability of the baseline module MO, which extrapolates LOFAR's TraP pipeline from the radio to the optical domain, is limited. Most queries write data, i.e. append or update, and therefore most of the time only one CPU core is being used. The MO crossmatching query is CPU-bound on all nodes and the full materialisation of the large intermediate results into memory introduces significant degradations of the performance, making it unfeasible to process the source lists within MeerLICHT's cadence time.
Smaller scans over sorted partitioned tables and less complex computations expose better instruction code locality and reduce intermediates for the M5 cross matching, from which the overall M5 pipeline performance distinctly benefits. The M5 cross-match query is about three orders of magnitude faster and behaves linearly. The queries in M5 utilise the available resources differently, since the cross-matching requires less CPU time. All queries are bound to maximum row numbers and finish execution within limited amounts of times. Internal database index structures further speed up the queries to run sublinear, giving the pipeline an additional boost of a factor of two. The partitioned database schema better predicts query behaviour on the long term, controls the overall pipeline performance and scales to larger numbers of sources.

\section{Conclusions}

High-cadence astronomical observatories have the potential to building up extremely large databases of catalogued sources and their light curves. Making scientific discoveries with the use of databases rely on the ability to efficiently grind the massive amounts of data. In this work, we matched optimised Big Data storage models to pipeline query access patterns in a layered storage system. The layers present the data in different formats, going from coarse high-level overviews at the top (all-sky) to the fine-grained details at the bottom tier (declination strip).

This work describes a scalable solution for the full-source database for the MeerLICHT and planned BlackGEM wide-field optical telescopes. We adopted the Transients Pipeline (TraP) database schema and pipeline queries from the LOFAR Transients Key Science Project as a baseline to ingest, process and store optical data from binary catalogue FITS files. We investigated the database schemas and query modules to optimise source cross matching and achieve long-term sublinear run times for all pipeline queries. We monitored all queries individually to study their long-term behaviour. Experiments with real data from the IPHAS Survey showed that the modified TraP baseline cross-match module is not scalable towards optical source densities. We developed an alternative cross-match module for database schema and query optimisations that improved the pipeline runs significantly. The column-oriented database schema design in which the data are partitioned horizontally according to declination strips allows the database to grow in size and simultaneously to run all pipeline queries in constant time, making this the preferred schema for processing large source lists at high cadence.

Cross-match algorithms of associating a new source list with the stored known sources are highly sensitive to their implementations. The list of known sources is reduced significantly by maintaining an up-to-date compact statistical sky model. After operations start, the model's size settles relatively early and therefore the cross-matching avoids large scans over continuously growing tables. We accelerated the cross-match positional lookups by three orders of magnitude with the use of MonetDB's default hash indexes on a single sorted column of declination values. The overall pipeline speed was increased by two orders of magnitude. Most queries run in constant time and only a few run in linear time with known upper limits.

In related tests, we noticed that the creation of a threedimensional tree index structure of the known catalogued sources becomes unbalanced after a while and that tree rebuilds are expensive, which slows down the pipeline run. This makes the $k \mathrm{~d}$ tree, where $k=3$ for the Cartesian co-ordinates, not the appropriate index structure in the sky model build-up phase, however, in a read-only static database it might well be the preferred index structure for fast positional look-ups and cross-matching. In this context, further Bkd tree performance investigations, especially 

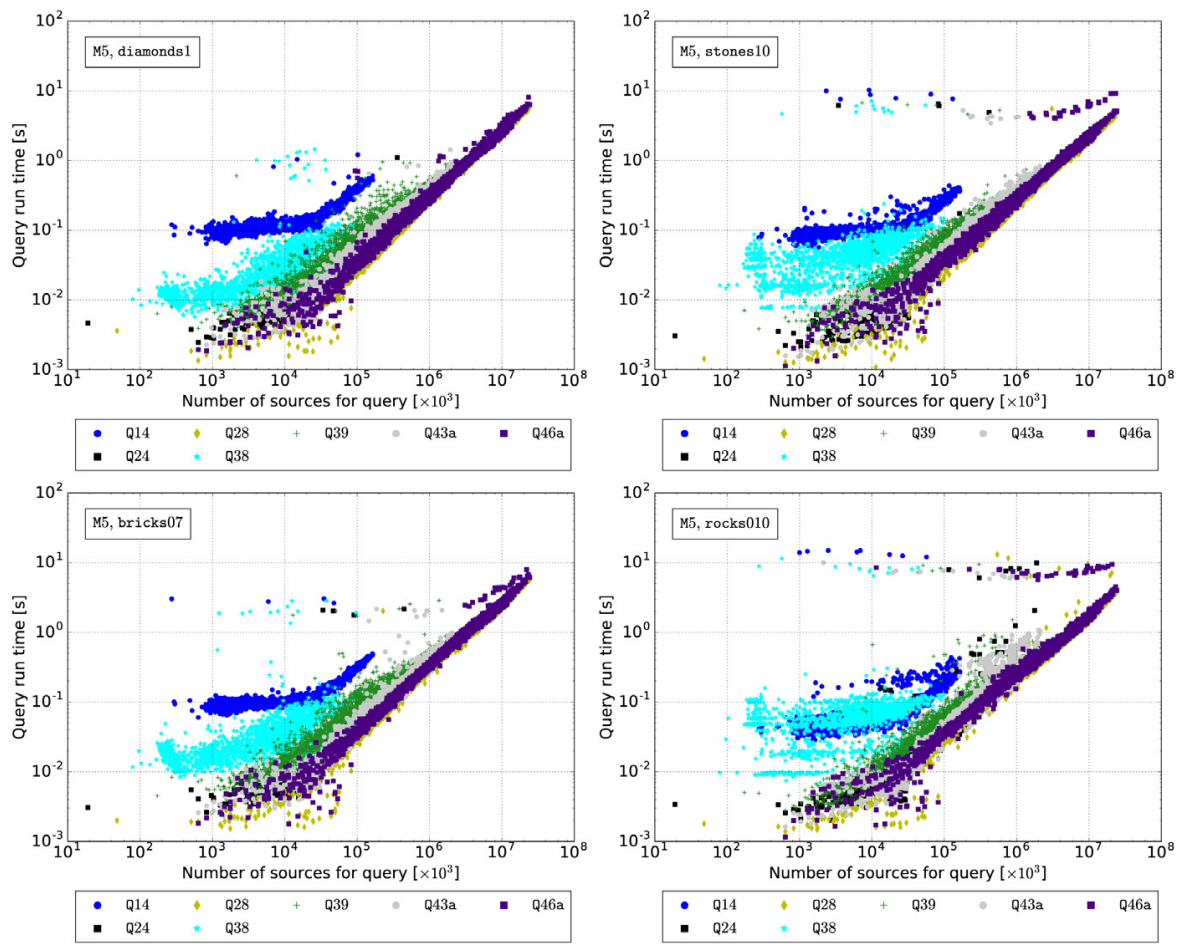

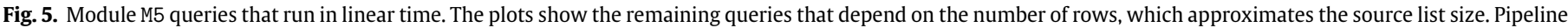
runs were on the different cluster nodes.

in the treatment of updating and removing points from the tree, optimising memory buffer sizes, number of trees in memory and tuning access to main memory may demonstrate its usefulness in dynamic, distributed, databases.

Performance results showed that the processing of IPHAS data, with similar average and peak source densities MeerLICHT will encounter, was feasible well within cadence limits of $25 \mathrm{~s}$. Source lists with average densities could be processed at rates of $5 \mathrm{~s}$ per image on nodes with modest CPUs and large RAM.

MeerLICHT's larger field of view implies larger source lists, but the sublinear-time behaviour and scalability of most queries, including the cross-matching, will not affect the performance. Queries that run in linear time will have similar performance for MeerLICHT, since the dependence scales with source density and not source list size for the most intense queries. The assumption of $12 \mathrm{~h}$ of observation per night and the offline mode that allows delays due to peaks will relax the criteria, meaning that with the alternative database and partitioning schema MeerLICHT fullsource data can be processed at the one minute cadence.

The BlackGEM array will consist of three telescopes, where each one will observe a different patch of the sky and will produce its own data stream, similar to MeerLICHT is doing. This simplifies parallelisation at an early stage, where the fields of view can be processed independently by multiple threads and database connections working on distinct partitions of the data. The in-memory column-oriented data storage structures of MonetDB match with the data ingestion and pipeline-specific queries. Independent parallel data streams will make the BlackGEM full-source pipeline capable of processing the data within its cadence time of one minute.

Development of the MonetDB database and the MeerLICHT \& BlackGEM full-source pipeline continues, where we will address improvements on data-partition querying, multi-dimensional tree indexing and techniques to visualise data in full-source archives.

\section{Acknowledgements}

We would like to thank the referee for the comments and suggestions that improved the paper. BS and MK acknowledge funding from the Dutch National Science Organisation NWO under Grant P4164 NWO project Big Bang Big Data ( 628-002-004 ).

\section{Appendix A. Insertion queries}

\section{A.1. I3, loading FITS data}

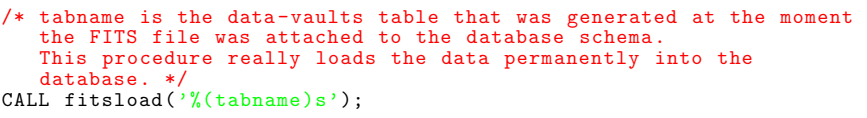

\section{A.2. I4, loading FITS header data}

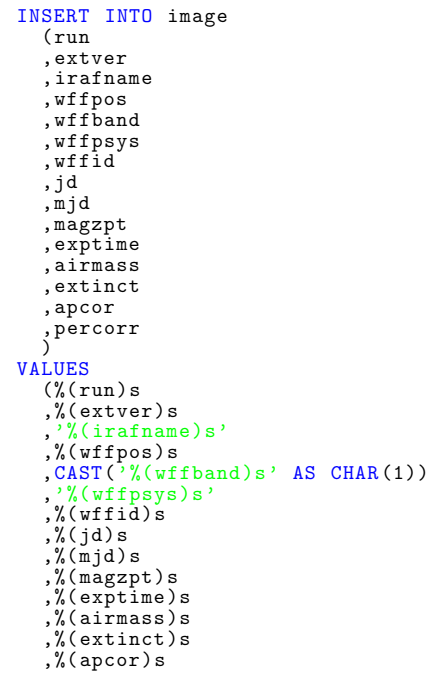


)$^{\%}(\operatorname{percorr}) \mathrm{s}$

\section{A.3. I5, inserting FITS data into permanent table}

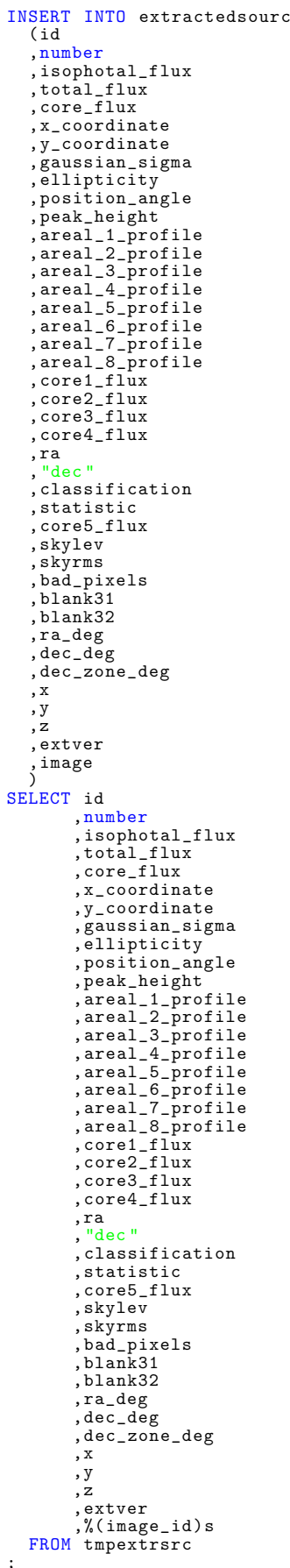




\section{Appendix B. Baseline cross-match module MO}

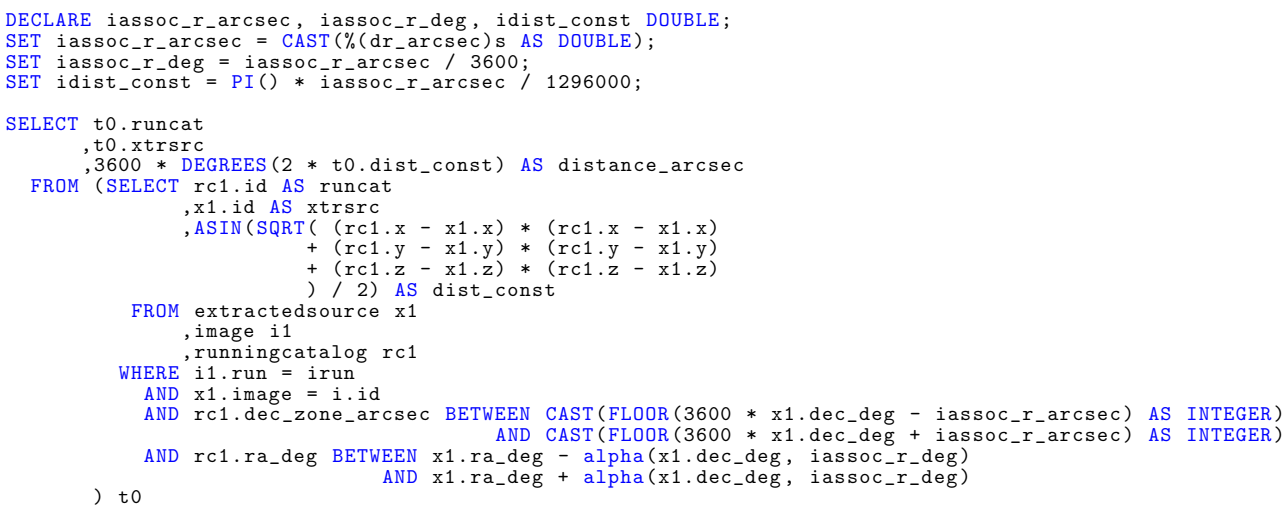

\section{Appendix C. Alternative cross-match module M5}

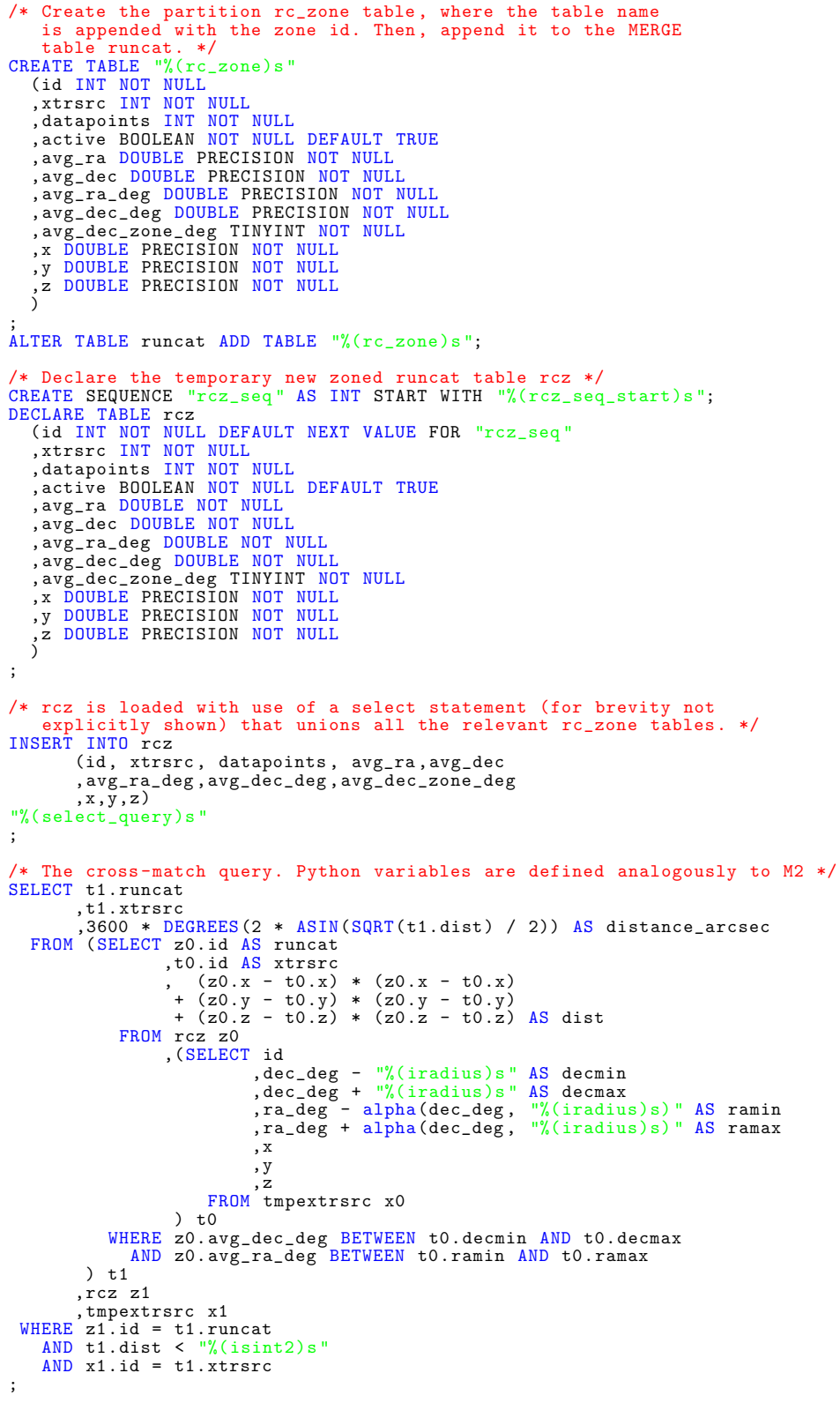




\section{References}

Abadi, D., Boncz, P., Harizopoulos, S., Idreos, S., Madden, S., 2012. The design and implementation of modern column-oriented database systems. Found. Trends Databases 5 (3).

Abadi, D.K., Madden, S.R., Hachem, N., 2008. Column-stores vs. row-stores: how different are they really? In: Proceedings of the 2008 ACM SIGMOD International Conference on Management of Data. ACM, pp. 967-980.

Alam, S., et al., 2015. Astrophys. J. Suppl. 219, 12A.

Barentsen, G., Farnhill, H.J., Drew, J.E., González-Solares, E.A., et al., 2014. Mon. Not R. Astron. Soc. 444, 3230B.

Becla, J., Wang, D.L., 2014. Enabling scalable data analytics for LSST and beyond, exascale radio astronomy. In: AAS Topical Conference Series, vol. 2. Proceedings of the conference held 30 March-4 April, 2014 in Monterey, California. Bulletin of the American Astronomical Society, Vol. 46, \#3, \#303.03, conf, 30303B.

Bloemen, S., Groot, P., Nelemans, G., Klein-Wolt, M., 2015. ASPC 496, 254B.

Boncz, P.A., May 2002. Monet: A Next-Generation DBMS Kernel for Query-Intensive Applications (Ph.D. thesis).Universiteit van Amsterdam.

Boncz, P.A., Manegold, S., Kersten, M.L., 1999. Database architecture optimized for the new bottleneck: Memory access. In: VLDB, Vol. 99. pp. 54-65.

Brederode, L.R., van den Heever, L., Esterhuyse, W., Jonas, J.L., 2016. SPIE 9906E, 25B.

Broekema, P.C., Van Nieuwpoort, R.V., Bal, H.E., June 2012. ExaScale high performance computing in the square kilometer array. In: Proceedings of the 2012 workshop on High-Performance Computing for Astronomy, AstroHPC'12, Delft, the Netherlands.

Cordier, B., Wei, J., Atteia, J.-L., Basa, S., Claret, A., Daigne, F., Deng, J., Dong, Y., Godet, O., Goldwurm, A., Götz, D., Han, X., Klotz, A., Lachaud, C., Osborne, J., Qiu, Y., Schanne, S., Wu, B., Wang, J., Wu, C., Xin, L., Zhang, B., Zhang, S.-N., 2015. PoS (SWIFT 10) 005.

Gray, J., Nieto-Santisteban, M.A., Szalay, A., 2006. The Zones Algorithm for Finding Points-Near-a-Point or Cross-Matching Spatial Datasets, Microsoft. Johns Hopkins University, MSR TR 200652.

Héman, , 2015. Updating Compressed Column-Stores (Ph.D. thesis).Vrije Universiteit, Amsterdam.

Ivanova, M., KargÄśn, Y., Kersten, M., Manegold, S., Zhang, Y., Datcu, M., Molina, D. 2013. Data vaults: a database welcome to scientific file repositories. In: SSDBM Baltimore, MD, USA.
Ivanova, M., Nes, N., Goncalves, R., Kersten, M., 2007. Monetdb/sql meets skyserver: the challenges of a scientific database. In: SSDBM. p. 13.

Ivezić, Ž, Connolly, A.J., Jurić, M., 2017. arXiv:1612.04772, http://dx.doi.org/10.1017/ S1743921316013156.

Juric, M., Tyson, T., 2015. LSST data management: Entering the era of petascale optical astronomy. HiA, 16, 675

Lazio, J.W., Kimball, A., Barger, A.J., Brandt, W.N., Chatterjee, S., Clarke, T.E., Condon, J.J., Dickman, R.L., Hunyh, M.T., Jarvis, M.J., Juric, M., Kassim, N.E., Myers, S.T., Nissanke, S., Osten, R., Zauderer, B.A., 2014. Radio astronomy in LSST era. PASP 126, 196L.

Manegold, S., Boncz, P., Kersten, M.L., 2002. Generic database cost models for hierarchical memory systems. In: VLDB.

Murphy, T., Chatterjee, S., Kaplan, D.L., Banyer, J., Bell, M.E., Bignall, H.E., Bower, G.C., Cameron, R.A., Coward, D.M., Cordes, J., et al., 2013. VAST: An ASKAP survey for variables and slow transients. PASA 30,6M.

Sidirourgos, L., Kersten, M.L., 2013. Column imprints: a secondary index structure. In: Proceedings of the ACM SIGMOD Conference on Management of Data, pp. 893-904.

Swinbank, John D., Staley, Tim D., Molenaar, Gijs J., Rol, Evert, Rowlinson, Antonia, Scheers, Bart, Spreeuw, Hanno, Bell, Martin E., Broderick, Jess W., Carbone, Dario, van der Horst, Alexander J., Law, Casey J., Wise, Michael, Breton, Rene P., Cendes, Yvette, Corbel, Stéphane, Eislöffel, Jochen, Falcke, Heino, Fender, Rob, Greißmeier, Jean-Mathias, Hessels, Jason W.T., Stappers, Benjamin W., Stewart, Adam J., Wijers, Ralph A.M.J., Wijnands, Rudy, 2015. Philippe Zarka the LOFAR transients pipeline. A\&C $11,25 S$.

Szalay, A.S., Blakeley, J.A., 2009. In: Hey, T., Tansley, S., Tolle, K. (Eds.), The Fourth Paradigm. Data-Intensive Scientific Discovery. Microsoft Corporation, USAi, p. 5.

Tingay, S.J., Goeke, R., et al., 2013. The murchison widefield array: the square kilometre array precursor at low radio frequencies. PASA 30, 7T.

van Haarlem, M.P., Wise, M.W., et al., 2012. LOFAR: The LOw-Frequency ARray. Astron. Astrophys. 2V, 556A.

York, D.G., et al., 2000. Astron. J. 120, 1579.

Zukowski, M., 2005. Hardware-conscious DBMS architecture for data-intensive applications. In: Proceedings of the 31st VLDB Conference, Trondheim, Norway. 